\title{
Fronteras y las relaciones civiles-militares en Colombia ${ }^{1}$
}

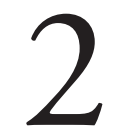

https://doi.org/10.21830/9789585318380.02

\author{
Manuel Ernesto Forero Garzón ${ }^{2}$ \\ Escuela Militar de Suboficiales "Sargento Inocencio Chinca”"
}

\section{Resumen}

La transformación de las amenazas a la seguridad internacional ha provocado un cambio en el paradigma de las relaciones cívico-militares, por ello, se hace absolutamente necesario dilucidar una política pública de fronteras, con fundamentos geopolíticos, como un instrumento dinamizador de las relaciones civiles-militares en Colombia, tarea que se aborda en el presente capítulo. Para ello, serán contrastados los enfoques de valor institucional y ocupacional de Moskos; la comprensión de la frontera como institución política primaria, según el pensamiento de Zapata-Barrero; y la teoría mixta en políticas públicas, de Roth, con la ubicación geográfica de aquellas unidades militares que tienen jurisdicción en los trece departamentos fronterizos del Estado colombiano. Todo lo anterior, tomando en cuenta que una política pública de fronteras de construcción mixta, donde la sociedad es parte activa en su formulación, conduce a que las Fuerzas Armadas flexibilicen la organización y transformen sus funciones, compartiendo valores y estrechando la comunicación con la sociedad. La presencia militar actual en los trece departamentos fronterizos permite la generación de conocimiento para comprender los riesgos, peligros y amenazas, tanto internas como externas, a la seguridad de Colombia, lo que resulta fundamental para una política de fronteras. En conclusión, la interacción de las relaciones civiles-militares mediante la planeación, diseño y ejecución de una política pública de fronteras, con base en fundamentos geopolíticos, es fuente de poder social para el Estado colombiano.

Palabras clave: relaciones civiles-militares; política pública; fronteras; geopolítica; Colombia.

1 Este capítulo hace parte de los resultados del proyecto de investigación "Relaciones civiles-militares en Colombia: una aproximación a los roles de las Fuerzas Militares” del Grupo de Investigación en Ciencias Militares de la Escuela Militar de Cadetes "General José María Córdova” categorizado en B por Minciencias y con código de registro COL0082556. Los puntos de vista y los resultados de este artículo pertenecen al autor y no reflejan necesariamente los de las instituciones participantes.

2 Profesional en Relaciones Internacionales y Estudios Políticos. Magíster en Relaciones Internacionales y doctorando en Seguridad Internacional (Universidad Nacional de Educación a Distancia-UNED). Profesor de la Unidad de Investigación Escuela Militar de Suboficiales "Sargento Inocencio Chincá” 


\section{Introducción}

Toda sociedad manifiesta una necesidad perdurable de crear un conjunto de valores, principios e instituciones para regular sus conductas e interrelaciones, y poder así, exigir la protección contra riesgos, peligros y amenazas de carácter interno y externo; es por esto que, las Fuerzas Armadas son una institución con valores y vocación de servicio hacia la sociedad que las origina. Para que un Estado pueda mantener su seguridad dispone de instituciones, recursos, normas, partidos políticos, entre otros elementos, pero cuando estos no resultan suficientes para que prevalezcan el interés y bienestar general, se hace necesario planear, diseñar y ejecutar políticas públicas orientadas a la transformación de la sociedad a la cual se debe defender y asegurar.

En Colombia, el paradigma de las relaciones civiles-militares ha sido transformado por la flexibilización de la agenda de la seguridad internacional del siglo XXI; donde la guerra no es la única amenaza, existe variedad de actores y se amplía la agenda de la seguridad, siendo caracterizada y definida por Bartolomé (2016) como amenazas trasnacionales y transversales, sumado a este cambio internacional, la situación sociopolítica de Colombia también ha cambiado sustancialmente en lo que respecta a las relaciones entre las Fuerzas Armadas y la sociedad colombiana. La conjugación de variables internacionales e internas convierten a las fronteras en punto de encuentro donde se dan cita fuerzas tangibles e intangibles que presionan a los Estados. Colombia comparte fronteras con doce Estados, donde toman parte trece departamentos y setenta y siete municipios, todos ellos con presencia y jurisdicciones de unidades militares de las Fuerzas Armadas colombianas. Paradójicamente, las zonas fronterizas se caracterizan por la falta de presencia del Estado y bajos niveles de desarrollo, en comparación con las zonas cercanas a la periferia del Gobierno central; uno de los factores que ha provocado esta desigualdad es la falta de una política pública de fronteras con fundamentos geopolíticos.

El presente capítulo ha sido motivado por la actual dinámica del sistema internacional, la importancia geopolítica de Colombia y su realidad interna; su objetivo principal consiste en dilucidar una política pública de fronteras con fundamentos geopolíticos, que sirva como un instrumento dinamizador 
de las relaciones civiles-militares en Colombia. El texto se desarrolla en tres partes: la primera de ellas, consta de un análisis acerca de cómo las doce fronteras (once establecidas mediante tratados internacionales, y una que resta por establecer con el Reino Unido) conforman un pilar fundamental para la formulación de una política pública, desde la perspectiva del enfoque teórico mixto (Estado-sociedad) de Roth (2002), que pueda contribuir a mantener la seguridad nacional. La segunda parte estudia la ubicación geográfica de las unidades militares ${ }^{3}$ de las Fuerzas Armadas de Colombia, con jurisdicción en los trece departamentos fronterizos, y realiza un contraste con el concepto de frontera como institución política primaria, al ser esta el punto de partida para la existencia de las demás instituciones del Estado, lo que ha demostrado el estudio de Zapata-Barrero (2012). Por último, las relaciones civiles-militares son abordadas desde el enfoque propuesto por Moskos (1991), que se fundamenta en los valores institucionales y ocupacionales de las Fuerzas Armadas que permiten una mejor comunicación entre estas y la sociedad; además, se hace un acercamiento a los riesgos, peligros y amenazas que afectan la seguridad de la nación para, finalmente, comprobar que las relaciones civiles-militares en Colombia son dinamizadas por una política pública de fronteras con fundamentos geopolíticos.

\section{Fronteras y geopolítica de Colombia: factores para una política pública}

Son varios los ejemplos difundidos en los diferentes medios de comunicación y redes sociales que reafirman la relación Estado, geopolítica, política de fronteras y seguridad. Entre ellos cabe mencionar la constante presión del Gobierno de los Estados Unidos para que México financie la construcción del muro en la frontera entre ambos países e impida el tránsito de migrantes centroamericanos y de otras nacionalidades hacia la potencia del norte. Otro

3 La ubicación geográfica y la jurisdicción de las unidades militares que serán relacionadas más adelante en el escrito, han sido tomadas de las páginas oficiales del Ejército Nacional de Colombia, de la Fuerza Área Colombiana y de la Armada de Colombia, información que has sido contrastada con la de los departamentos fronterizos. 
ejemplo es el muro entre Israel y Palestina, que a diario presenta perturbaciones que afectan la seguridad a ambos lados de la frontera. El Conflicto Armado Internacional en Siria es otro ejemplo del poder político de las fronteras, en este caso se involucran Estados fronterizos de Siria (Turquía e Israel) y Estados más allá de sus fronteras (Irán y Arabia Saudita) que dentro de sus cálculos políticos consideran al conflicto una amenaza a su seguridad. Por último, en la actualidad la Federación Rusa y Ucrania mantienen una disputa fronteriza por la anexión de la Península de Crimea por parte de Rusia y su influencia en los territorios de la frontera terrestre entre los dos Estados. Estos ejemplos de la dinámica actual del sistema internacional dejan en evidencia una conjunción de variables que involucra Estados, la delimitación de sus fronteras y su seguridad.

No se puede concebir la existencia de un Estado sin una base territorial y un espacio geográfico, y en los cuales converge un conglomerado humano con identidades e intereses propios, que busca protegerse de riesgos, peligros y amenazas de carácter interno y externo, esto conduce, necesariamente, a que el Estado planee, diseñe y ejecute políticas públicas para la protección y el bienestar de su población, así como para el afianzamiento de valores que identifiquen a la nación. A lo anterior Reynolds (1977) lo denomina "interés nacional”. Por su parte, Morgenthau (1986), en su análisis sobre el poder nacional de los Estados, establece dos elementos que son relativamente estables: geografía y recursos naturales, y otros que están sometidos a constantes cambios: capacidad industrial, aspectos militares, población, carácter nacional, moral nacional, calidad de sus diplomáticos y capacidad de la clase gobernante. El espacio geográfico está íntimamente relacionado con el accionar del Estado y la protección de su población, es por tal razón que las fronteras son tan importantes para cualquier Estado, pues representan la máxima expresión del poder político y soberano.

Colombia, por su posición geográfica, se encuentra ubicada estratégicamente en el subcontinente latinoamericano, que comprende zonas geográficas de América Central, El Caribe, y América del Sur. En este sentido, y según la jerarquización del mundo por regiones postulada por Cohen (2003) regiones geoestratégicas, geopolíticas y estratégicas - Colombia se halla en la 
región geoestratégica del mundo marítimo dominada por Estados Unidos ${ }^{4} \mathrm{y}$ en la región geopolítica de las Américas ${ }^{5}$. Al traslapar la jerarquía del mundo y la importancia geopolítica de Colombia se obtiene como resultado una proyección al Asia-Pacífico, epicentro del comercio internacional y disputa del poder mundial, otro factor de importancia para el Estado colombiano en este océano, es la presencia en la Antártida por la ubicación geográfica de la Isla de Malpelo. La importancia geopolítica en el Mar Caribe se sustenta por el canal de Panamá, presencia de rutas comerciales, transporte de petróleo, actividades de narcotráfico y la prolongación al Océano Atlántico, Europa y África. Finalmente, la importancia geopolítica de Colombia con las cuencas hidrográficas de la Orinoquía y la Amazonia que la conectan la cuenca del Río de la plata.

Apelando una vez más a los postulados sobre geopolítica de Cohen, Colombia comparte fronteras con doce Estados ${ }^{6}$ donde se comprometen la soberanía y seguridad de trece departamentos ${ }^{7}$ y setenta y siete municipios. Según el Instituto Geográfico Agustín Codazzi, Colombia cuantitativamente posee una superficie total de $2.070 .408 \mathrm{~km}^{2}$ compuesta por una extensión territorial de $1.141 .749 \mathrm{~km}^{2}$, que equivale al $55.14 \%$ de la superficie total y un área marítima de $928.660 \mathrm{~km}^{2}$ que corresponde al 44.9\%, y que integran a 32 departamentos y 1123 municipios.

Molano-Rojas y Salazar (2016) realizan un análisis detallado de las fronteras de Colombia. Los trece departamentos fronterizos representan el 39,6\% y los setenta y siete municipios el 9,2\% de la población total de Colombia. De otro lado, estas entidades territoriales albergan el 70\% de los resguardos indí-

4 Este dominio está siendo cuestionado y debilitado por la denominada "Ruta de le Seda Marítima de China”, que mediante su política exterior es dueña, socia y participante en los puertos marítimos estratégicos para el comercio internacional. Adicionalmente, posee la mayor flota mercante del mundo y está aplicando un plan te renovación y actualización de su Armada.

5 Cohen afirma que la inmadurez geopolítica de esta región es asumida por Brasil.

6 De los doce Estados con que se comparte frontera, once se han establecido mediante tratados internacionales o instrumentos del Derecho Internacional Público (Brasil, Costa Rica, Ecuador, Haití, Honduras, Jamaica, Panamá, Perú, Nicaragua, República Dominicana y Venezuela). Y una frontera a determinar con el Reino Unido por la cercanía de la isla de Gran Caimán y el Departamento de San Andrés, Providencia y Santa Catalina.

7 La Guajira, Cesar, Norte de Santander, Arauca, Guainía, Vichada, Boyacá, Vaupés, Amazonas, Nariño, Chocó, Putumayo, y San Andrés, Providencia y Santa Catalina. 
genas y el $71 \%$ de titulaciones colectivas de comunidades afrodescendientes. Además, estos autores demuestran que los departamentos fronterizos se caracterizan por una baja participación en el PIB, mayor índice de desempleo, alto grado de necesidades insatisfechas, poca cobertura en educación superior, reducida conexión a la red nacional de energía eléctrica, precios de combustibles altos en relación al otro lado de la frontera, poca capacidad productiva para la exportación, zonas sensibles y vulnerables para la seguridad y defensa, acceso limitado a las tecnologías de la comunicación, departamentos fronterizos aislados de las vías de comunicación, el único departamento que está interconectado con el interior del país es Boyacá, y por último, mínima generación de recursos propios, pues estos departamentos dependen de la asignación del Gobierno central.

Para Zapata-Barrero las fronteras son una institución primaria que da origen a las demás políticas del Estado, así que sin una política pública de fronteras no pueden existir las otras políticas (2012, pp. 49-51). Las políticas de defensa y seguridad nacional del pasado ${ }^{8}$ coinciden con las del actual Gobierno ${ }^{9}$, pues no definen de forma clara y precisa las amenazas externas ${ }^{10}$ que afectan o perturban el interés nacional de Colombia. Tampoco se sostienen sobre un fundamento geopolítico, y no se preocupan por el hecho de que el Estado esté prácticamente ausente en las regiones más apartadas; lo que reafirma, una vez más, la inexistencia de una política pública de fronteras con bases geopolíticas; con ello, tácitamente, se acepta que no existe una plena soberanía por parte del Estado y se reconoce la pérdida del control de las fronteras (Zapata-Barrero, 2012, p. 44).

Asimismo, el documento CONPES11 3805/2014: "Prosperidad para las fronteras de Colombia" define la política de fronteras y fija como objetivo

8 Política Integral de Seguridad y Defensa para la Prosperidad (2011-2018) del Gobierno de Juan Manuel Santos, que es una continuidad de la Política de Seguridad Democrática del Gobierno de Álvaro Uribe Vélez.

9 Política de Defensa y Seguridad para la Legalidad, el Emprendimiento y la Equidad (2019) del Gobierno de Iván Duque.

10 Las amenazas enunciadas son: migración masiva, regímenes no democráticos, crisis humanitarias, pretensión de despojo de territorio y tráfico ilícito de migrantes, armas, municiones, drogas y pesca ilegal.

11 Consejo Nacional de Política Económica y Social 
generar fronteras más incluyentes, sostenibles y seguras. Describe la tipología de las fronteras, menciona los 13 departamentos y 77 municipios fronterizos, hace un diagnóstico social, político y económico de estas regiones en comparación con las del resto del país y, finalmente, concluye que las zonas fronterizas son las menos desarrolladas del Estado colombiano. Cabe resaltar que el documento estima que estos departamentos fronterizos poseen ventajas comparativas de gran valor geopolítico y geoestratégico, pero se vuelve al común denominador que denota la incapacidad de poder formular una política pública de fronteras que demuestre un estudio geopolítico de Colombia. Se echa de menos una identificación de las amenazas internas y externas que afectan el interés nacional, y que se entienda que una política de seguridad y defensa nacional no debe responder a ideologías de partido o del gobierno de turno, sino que obedece a la búsqueda del bien común, es decir, a un objetivo político de Estado y no de gobierno.

El Plan Nacional de Desarrollo (2018-2022) no articula en sus pactos de defensa nacional, política exterior ni zonas de productividad (regiones de San Andrés, Amazonia, Orinoquía, Santanderes y océanos), un fundamento geopolítico como sustento de una política de fronteras. Es más, en el Plan se acepta que no existe en Colombia una política de fronteras, y que la política exterior colombiana tiene como propósito formular una política de fronteras y de soberanía territorial (DNP, 2018, pp. 116-117).

Ahora bien, se puede afirmar que existe una brecha entre el ideal de una frontera y la realidad de estas zonas, es decir, el Estado colombiano aún se sigue enfrentando a una problemática sin resolver. Cuervo (2014) afirma que en Colombia hablar de la implementación de política pública no es más que un eufemismo, pues esta se confunde con programas de gobiernos y planes de inversión (p. 144). Roth (2002) considera, en su estudio de las políticas públicas, que el Estado se enfrenta a problemáticas de toda índole por su incapacidad de penetrar todos los espacios sociales, más aún en las zonas de periferia, lo que conduce a una estrategia legítima de movilización del Estado y la sociedad para dar origen a una política pública (pp. 22-25). 


\section{Ubicación geográfica de unidades militares}

Un común denominador en las relaciones civiles-militares es la subordinación del poder militar al poder civil de la sociedad, así como al cumplimiento de lo establecido en la Constitución Política. Tanto la funcionalidad y como la finalidad constitucional de las Fuerzas Armadas están regidas en Colombia por el capítulo séptimo, en especial los artículos 216 y 217, de la Constitución Política de Colombia de 1991. Estos referentes legales determinan que la defensa de la soberanía, la independencia, la integridad territorial y el orden constitucional de Colombia recaen sobre sus Fuerzas Armadas, proyectando el accionar de estas hacia el interior del Estado y frente las posibles acciones externas que pretendan modificar negativamente estos fines.

Históricamente, las Fuerzas Armadas en Colombia se han empleado para contrarrestar el conflicto armado no internacional, provocando que la ubicación geográfica y la jurisdicción de estas se concentre en aquellas regiones del país donde las condiciones de orden público exijan la presencia militar para contener, debilitar y combatir a los grupos armados que están por fuera del monopolio de la fuerza ejercida por el Estado, restando importancia a las zonas de frontera, lo que las hace vulnerables a riesgos, peligros y amenazas internas y externas que atentan contra su seguridad.

A este respecto, en su análisis sobre los intereses geopolíticos de Colombia, Esquivel (2015) propone que el Estado debe asumir un liderazgo en sus áreas de influencia del Pacífico occidental, la cordillera de los Andes, la Amazonia, el istmo centroamericano, el Caribe y la Orinoquía, donde la clase dirigente tiene que orientar su accionar hacia estas regiones estratégicas, para evitar la pérdida de territorio y ejercer poder estatal. En esta misma dinámica de fronteras y ejercicio del poder, Quintero y Forero (2018), en su estudio de los libros blancos de defensa del Brasil, Perú y la incidencia de estos para Colombia, proponen desarrollar códigos geopolíticos locales y regionales que involucren el factor fronteras, para defender y asegurar a la nación colombiana contra riesgos, peligros y amenazas tanto internas como externas.

Las dos apreciaciones anteriores (intereses geopolíticos de Colombia y códigos geopolíticos) resaltan la importancia estratégica de los trece departamentos fronterizos de Colombia, en esta lógica de la geopolítica, estas zonas de frontera deberían contar con mayor presencia del Estado, niveles óptimos 
de crecimiento y desarrollo, una sólida infraestructura y estrechos lazos de cooperación internacional. Sin embargo, la realidad sociopolítica demuestra que estas áreas geográficas son las más sensibles ante las amenazas transnacionales y transversales, así como frente al conflicto armado no internacional aún presente en el país. En este punto cabe recordar que las Fuerzas Armadas hacen parte de la institucionalidad del Estado, el cual les asigna funciones específicas para dar cumplimiento al mandato constitucional; para alcanzar tal objetivo, las unidades militares son desplegadas y proyectadas en el territorio nacional con la asignación de jurisdicción sobre departamentos y municipios. La tabla 1 muestra la ubicación geográfica de esas unidades en los departamentos fronterizos de Colombia.

Tabla 1. Unidades militares en departamentos fronterizos de Colombia

\begin{tabular}{|c|c|c|}
\hline Estados fronterizos & Departamentos & $\begin{array}{l}\text { Unidades militares con presencia y } \\
\text { proyección en departamentos fronterizos }\end{array}$ \\
\hline Venezuela & $\begin{array}{l}\text { La Guajira, Cesar, Norte } \\
\text { de Santander, Arauca, } \\
\text { Guainía, Vichada y Boyacá }\end{array}$ & $\begin{array}{l}\text { X Brigada blindada, Comando Conjunto } \\
\text { del Caribe, Brigadas XXX, V, I, XIV, } \\
\text { XVI, XXVIII y XVIII, Fuerza de Tarea } \\
\text { Quirón, Grupo Aéreo Vichada y Brigada } \\
\text { Fluvial No. } 1 .\end{array}$ \\
\hline Brasil & $\begin{array}{l}\text { Guainía, Vaupés y } \\
\text { Amazonas }\end{array}$ & $\begin{array}{l}\text { Brigadas de Selva XXXI y XXVI, Brigada } \\
\text { XXVIII y Grupo Aéreo Amazonas. }\end{array}$ \\
\hline Perú & Amazonas y Putumayo & $\begin{array}{l}\text { Brigadas de Selva XXVI y XXVII y } \\
\text { Brigada fluvial No. } 1 .\end{array}$ \\
\hline Ecuador & Putumayo y Nariño & $\begin{array}{l}\text { Brigadas III y XXIII, Brigada de Selva } \\
\text { XXVII, Fuerza Naval del Pacífico y } \\
\text { Brigada fluvial No. } 2 .\end{array}$ \\
\hline Panamá & Chocó & $\begin{array}{l}\text { Brigada de Selva XV, Fuerza de Tarea } \\
\text { Conjunta Titán y Fuerza Naval del } \\
\text { Pacífico. }\end{array}$ \\
\hline $\begin{array}{l}\text { Estados del Mar } \\
\text { Caribe (Nicaragua, } \\
\text { Honduras, Costa } \\
\text { Rica, Haití, República } \\
\text { Dominicana, y } \\
\text { Jamaica) }\end{array}$ & $\begin{array}{l}\text { San Andrés, Providencia y } \\
\text { Santa Catalina }\end{array}$ & $\begin{array}{l}\text { Comando Aéreo No. 3, Grupo Aéreo } \\
\text { San Andrés, Fuerza Naval del Caribe y } \\
\text { Comando Específico de San Andrés y } \\
\text { Providencia. }\end{array}$ \\
\hline
\end{tabular}

Fuente: elaboración propia con la información de ubicación geográfica y jurisdicción de las unidades militares de las Fuerzas Armadas de Colombia que hacen presencia en los trece departamentos fronterizos. 
Una revisión cuantitativa de las unidades militares con presencia en los departamentos fronterizos deja como resultado que la frontera con Venezuela (2219 km, la más extensa de Colombia) involucra a siete departamentos, con presencia de once unidades militares de las Fuerzas Armadas, siendo la mayor presencia militar en comparación con los otros Estados con los que comparte frontera. Lo anterior encuentra su explicación en el diferendo actual por la delimitación de la frontera en el departamento de La Guajira, por las constantes migraciones de ciudadanos venezolanos, presencia de crimen organizado y las diferencias políticas e ideológicas entre los gobiernos de Colombia y Venezuela.

Con la comparación de la presencia de unidades militares en zonas fronterizas no se pretende afirmar que esa sea la única frontera estratégica y sensible para Colombia. Sus fronteras terrestres se caracterizan por ser extensas, de difícil acceso y de topografía atípica, lo que dificulta su control y las hace propicias para el desarrollo de actividades ilegales que afectan la condición de seguridad del Estado colombiano. Una característica de las relaciones civiles-militares es la flexibilización de funciones para Fuerzas Armadas, y que, al interactuar en estas zonas de fronteras les permite conocer, afrontar y contrarrestar lo que Bartolomé (2002, pp. 159-220) caracterizó como amenazas transnacionales y transversales ${ }^{12}$.

En la actualidad, las unidades militares acantonadas en los departamentos fronterizos enfrentan amenazas transnacionales de migración masiva por la complejidad y naturaleza de la frontera, lo que se ha agravado con la pandemia del COVID-19, que representa una amenaza transversal que ya ha afectado a los departamentos del Amazonas, Nariño y Norte de Santander. Otras amenazas transnacionales ${ }^{13}$ son el crimen organizado y el tráfico de armas livianas, beneficiados por la poca presencia del Estado, las comunidades vulnerables y la topografía agreste de la región. Las unidades militares también se enfrentan al deterioro ambiental por la extracción, tráfico y comerciali-

12 Son considerados como factores transversales la pobreza, las pandemias y la corrupción. Todos ellos debilitan al Estado y la democracia.

13 Las otras amenazas mencionadas, caracterizadas y explicadas por Bartolomé, son: el terrorismo internacional, las armas de destrucción masiva y la guerra electrónica, siendo esta última la única amenaza nueva en la agenda de la seguridad internacional. 
zación de recursos estratégicos de manera ilegal, como oro, madera, coltán, entre otros, afectando el medioambiente de comunidades raizales y trayendo consigo consecuencias sociales como el desplazamiento forzado, la violencia de género, la persecución y el asesinato de líderes sociales.

Sumado a las amenazas transnacionales y transversales, las unidades militares asumen la responsabilidad de enfrentar a grupos armados organizados, propios de un conflicto armado no internacional, y a bandas criminales, organizaciones ilegales que desarrollan actividades de narcotráfico, actos terroristas, secuestro y extorsión.

Objetiva y subjetivamente, las unidades militares conocen las regiones menos desarrolladas de Colombia, enfrentan una variedad de riesgos, peligros y amenazas internas y externas que afectan su seguridad, conviven con las comunidades más vulnerables de las zonas fronterizas, son un puente de comunicación entre las necesidades de la región y el Estado colombiano, representan el primer escudo para defender y asegurar a la nación y constituyen la institución estatal de mayor presencia en los departamentos fronterizos. En este orden de ideas, las relaciones civiles-militares flexibilizan el paradigma tradicional de las relaciones civiles y militares, donde cada institución mantenía su independencia, funcionalidad, valores y principios.

\section{Relaciones civiles-militares, política pública y fronteras}

A pesar de que la capacidad militar no posee la misma transcendencia de años anteriores en la agenda de la seguridad, y de que existen naciones que no cuentan con Fuerzas Armadas dentro de tu aparato institucional, el factor militar sigue siendo un poder nacional de los Estados. Mann (1991) considera que este poder militar es una fuente de poder social. Por su parte, Morgenthau le asigna cuatro variables fundamentales: tecnología, liderazgo, cantidad y calidad, necesarias para cumplir con las tareas que le son encomendadas a las Fuerzas Armadas (1986, pp. 156-160). La evolución y consolidación de la seguridad internacional contemporánea ha demostrado que la guerra no es la única amenaza a la que se enfrentan los Estados, razón por la cual se ha incor- 
porado otro listado de riesgos, peligros y amenazas, los cuales deben ser enfrentados desde un enfoque multidimensional. Bartolomé (2016) sostiene que la anterior dinámica influye en el replanteo de la seguridad nacional, otorgándole un valor fundamental a la supervivencia del Estado y la integridad territorial, e incorporando los ámbitos interno y externo (p. 124).

Así las cosas, el paradigma de las relaciones civiles-militares proporciona un escenario sociopolítico para que la sociedad sea un actor más activo en lo referente a la defensa y la seguridad nacional. El modelo de relaciones civiles-militares y el perfil del militar posmoderno de Moskos (1991) podrían analizarse desde las ópticas de la organización, la funcionalidad y las relaciones. Desde lo organizacional es una institución flexible y transformada que sigue subordinada al control y poder político, con niveles de profesionalismo que denota constante capacitación e igualdad de género, y que incorpora a civiles expertos para comprender, analizar y elaborar la defensa. La funcionalidad del militar es una vocación de servicio en procura del bienestar general sobre el particular, característica propia de un Estado social de derecho, donde prima la dignidad del ser humano. Por lo anterior, la defensa y seguridad del Estado son bienes públicos cuya protección recae en las Fuerzas Armadas, encargadas de enfrentar amenazas internas y externas que implican un abanico de misiones que deben ser cumplidas. Por último, lo militar y lo civil encuentran un escenario propicio de interacción y comunicación mejorada al compartir valores y responsabilidades en una sociedad vulnerable, que exige un diálogo con el Estado para ser escuchada. Tal y como señala Moskos, el Estado, la sociedad y las Fuerzas Armadas se fusionan para enfrentar fuerzas internas que arriesgan la convivencia y el orden constitucional - y fuerzas externas, estatales y no estatales, — que transcienden las fronteras amenazando el libre y normal desarrollo de una nación-.

La ubicación y jurisdicción de las unidades militares de las Fuerzas Armadas de Colombia en los departamentos fronterizos expresan relaciones socio-espaciales-políticas con presencia de actores de la sociedad civil y actores militares, que, independientemente de su naturaleza disímil, son convocados para estudiar, analizar, planear y enfrentar las amenazas que afectan el bienestar de la sociedad. 
En lo social, las Fuerzas Armadas colombianas, dentro la flexibilización organizacional y apelando al enfoque de Moskos, conviven con las necesidades y problemáticas de las zonas fronterizas, tanto así que las entidades territoriales de departamentos y municipios convocan de forma permanente a comandantes y representantes de las unidades militares a participar en los consejos comunales de seguridad y convivencia, donde se exponen y discuten todos los riesgos, peligros y amenazas a la seguridad de esas zonas.

Otro factor de las relaciones sociales es la igualdad de sexo, aspecto que se ha integrado también al ámbito militar, razón por la cual en la actualidad es permitido el ingreso de mujeres a escuelas de formación de oficiales y suboficiales, caracterizándose por la simetría en las prestaciones legales vigentes.

Por último, enfrentarse a algunas de las amenazas transnacionales y transversales requiere la incorporación de personal técnico, profesional y académico, pues resulta fundamental una evaluación interdisciplinar para enfrentar cada una de las amenazas que afectan el interés nacional.

En lo que respecta a las relaciones espaciales, la presencia militar en los departamentos fronterizos, cuya valoración estratégica y geopolítica no es asumida totalmente desde el Gobierno central, ratifica que las Fuerzas Armadas colombianas están comprometidas con el esquema contemporáneo de la seguridad, pero no logran alcanzar los niveles óptimos de desarrollo, lo que conduce necesariamente a la presencia de más instituciones del Estado para hacer frente a las problemáticas de estas regiones de la periferia. Las relaciones políticas se pueden abordar desde dos perspectivas: poder y bienestar común. Así, las Fuerzas Armadas colombianas mantienen el monopolio de la fuerza, y harán uso del poder coercitivo cuando lo constitucional sea exigido, dentro de los parámetros del Derecho Internacional de los Derechos Humanos, el Derecho Internacional Humanitario y la normatividad interna de Colombia. Por otra parte, con relación al bienestar común en las zonas de frontera, sociedad y Fuerzas Armadas interactúan para optimizar recursos asignados por el Estado, en procura de logar el fin último de todo Estado social de derecho.

La presencia y el ejercicio de las unidades militares de jurisdicción fronteriza no son suficientes para que estas zonas geográficas alcancen los niveles de crecimiento, desarrollo y seguridad de otras regiones, lo que hace necesaria la 
participación de más instituciones estatales para cumplir con la exigencia de la seguridad contemporánea, que implica reaccionar con múltiples dimensiones ante amenazas al bienestar común de la nación, hecho que demuestra la necesidad de una política pública de fronteras con fundamentos geopolíticos.

Para Roth (2002), todo Estado — sin importar su régimen político e ideológico, la tipología de su economía ni sus niveles de desarrollo- enfrenta a diario problemáticas tradicionales y nuevas, internas y externas, que afectan el desarrollo normal de la nación, las cuales se manifiestan a través de los canales de comunicación entre este y la sociedad, que exige el deber institucional de brindar garantías para tener una calidad de vida digna. La realidad sociopolítica influye sobre el funcionamiento del Estado al no poder permear todos los espacios sociales, especialmente en las zonas de la periferia, provocando cambios institucionales. Un ejemplo de ello es la formulación de políticas públicas que obliga al Estado a un sistema complejo de acciones, movilización de actores, tanto públicos como privados, donde cada uno persigue intereses contradictorios pero armonizables para el bienestar común de la sociedad (pp. 17-41).

A este respecto, Roth considera que para transformar a una sociedad la política pública tiene elementos esenciales, como la implicación del Gobierno, la percepción del problema, y la definición de objetivos y procesos. Para alcanzar dicha transformación este autor propone tres enfoques teóricos relacionados con el Estado y las políticas públicas: uno centrado en la sociedad; otro en el Estado; y, finalmente, uno mixto - en un punto intermedio entre los dos primeros- (2002, pp. 27-29). Para efectos del presente capítulo, se hace énfasis en el último enfoque, pues el enfoque mixto plantea que la sociedad y el Estado, más que tener relaciones, se interrelacionan, es decir que interactúan en un plano horizontal, de este modo desaparece la interacción vertical, que implica el dominio de uno sobre otro, y se privilegia la interrelación entre los ámbitos de lo público y lo privado (2002, pp. 31-32).

Una política pública de fronteras con fundamentos geopolíticos mantiene y reactiva las relaciones civiles-militares, porque implica al Gobierno y a todos los sectores de la sociedad en la tarea de evaluar cuál es el interés nacional afectado por los riesgos, peligros y amenazas, tanto internas como externas, que perjudican la condición de la seguridad nacional. Es por lo anterior que, 
la presencia de las unidades militares colombianas en zonas fronterizas de valor geopolítico son fuentes de información primaria y secundaria que el Gobierno central y demás entidades territoriales de Colombia deben considerar para la planeación, diseño y formulación de políticas públicas que englobe a la frontera como una institución política primaria. Finalmente, las unidades militares de estas zonas están en la capacidad de formular objetivos y procesos interrelacionados con la sociedad, pues conviven con las comunidades de estas regiones, por ello resulta sumamente importante su participación y comunicación constante con el Gobierno central.

\section{Conclusiones}

Las condiciones objetivas para la construcción en Colombia de una política pública de fronteras con fundamentos geopolíticos están dadas: el país posee una ubicación geográfica estratégica y de importancia geopolítica; se encuentra inmerso en la dinámica del sistema internacional; posee trece departamentos fronterizos que se encuentran presionados por las amenazas transnacionales transversales y las acciones propias del conflicto armado no internacional, caracterizándose por bajos niveles de desarrollo en comparación con el resto de las zonas más próximas al gobierno central. Por otra parte, existen condiciones subjetivas como la incapacidad estatal para movilizar recursos, actores e instituciones públicas y privadas, lo que impide consolidar a la frontera como una institución política primaria.

Tomando en cuenta tanto las condiciones objetivas como las subjetivas, las unidades militares fronterizas de las Fuerzas Armadas de Colombia son protagonistas de la realidad sociopolítica, nacional e internacional, y se interrelacionan con la sociedad para cumplir la misión constitucional que las faculta para adelantar operaciones y acciones de múltiples tipologías.

Entonces, una política pública de fronteras con fundamentos geopolíticos fusiona a todas las instituciones del Estado, a todos los actores de la sociedad y a la cooperación internacional. En este contexto, los perfiles de las relaciones civiles-militares y el militar posmoderno deben flexibilizar los valores institucionales y organizacionales, compartir principios y responsabilidades con la 
sociedad, e incorporar personal civil con experiencia en el sector de defensa y seguridad.

A modo de reflexión final, una política pública con las características anotadas puede llegar a constituir un dinamizador de las relaciones civiles-militares en Colombia, donde el punto de convergencia entre el poder militar y la sociedad se dé en las interrelaciones socio-espacial-politicas que abordan las necesidades, problemáticas y amenazas a la seguridad nacional, las zonas geográficas de importancia geopolítica, el poder coercitivo dentro de los parámetros legales nacionales e internacionales, además del bienestar común de toda la población.

\section{Referencias}

Armada de Colombia. (2020). Armada Nacional. Armada de Colombia. https://www.armada. mil.co

Bartolomé, M. (2006). La seguridad internacional en el siglo XXI, más allá de Westfalia y Clausewitz. Academia Nacional de Estudios Políticos y Estratégicos, Ministerio de Defensa Nacional.

Bartolomé, M. (2016). Algunas aproximaciones a la agenda de la Seguridad Internacional contemporánea y la influencia teórica en sus contenidos. Revista Politica y estrategia, (128), 101-134. http://www.politicayestrategia.cl/index.php/rpye/article/view/85/177

Cohen, S. (2003). Geopolitics of the world system. MD.: Rowman \& Littlefield.

Cuervo, J. (2014). La implementación de política pública: un problema conceptual y metodológico. En C. Holguín (Ed.), Politicas públicas sociales y territoriales: reflexiones teóricas y estudios de casos (pp. 139-159). Universidad Autónoma de Occidente.

Departamento de Planeación Nacional. (2014). Documento CONPES 3804 Prosperidad para las fronteras de Colombia. Departamento de Planeación Nacional.

Departamento de Planeación Nacional. (2018). Plan Nacional de Desarrollo (2018-2022). Departamento de Planeación Nacional.

Ejército Nacional de Colombia. (2020). Ejército Nacional de Colombia. Ejército Nacional de Colombia: https://www.ejercito.mil.co

Esquivel, R. (2015). Intereses geopolíticos de Colombia: Estudios en Seguridad y Defensa, 10(19), 71-86. https://www.esdeguerevistacientifica.edu.co/index.php/estudios/article/view/69

Fuerza Aérea Colombiana. (2020). Fuerza Aérea Colombiana. Fuerza Aérea Colombiana. https:// www.fac.mil.co

Gobierno de Colombia. (1991) Constitución Politica. Legis.

Mann, M. (1991). Las fuentes del poder social. I. Alianza Editorial. 
Ministerio de Defensa Nacional. (2011). Política Integral de Seguridad y Defensa para la prosperidad. Ministerio de Defensa Nacional.

Ministerio de Defensa Nacional. (2019). Política de Defensa y Seguridad para la legalidad, el emprendimiento y la equidad. Ministerio de Defensa Nacional.

Molano-Rojas, A., \& Zarama, F. (2016). Fronteras: gobernanza, sensibilidad y vulnerabilidad. En I. Olózaga, Fronteras en Colombia como zonas estratégicas: Análisis y perspectivas (pp. 16-47). Andrés Molano-Rojas.

Morgenthau, H. (1986). Politica entre las naciones. La lucha por el poder y la paz (6a ed.). Grupo Editor Latinoamericano.

Moskos, C. (1991). Tendencias institucionales y ocupacionales en las Fuerzas Armadas. En C. Moskos, \& F. Wood (Coomp.), Lo militar: ¿más que una profesión? Ministerio de Defensa.

Quintero, S., \& Forero, M. (2018). Los libros blancos de defensa de Perú y Brasil: aspectos geopolíticos, seguridad regional e incidencia para Colombia. En C. Álvarez, \& A. Fernández-Osorio (Eds.), Hacia una gran estrategia en Colombia: Construcción de politica pública en seguridad y defensa. Volumen 3: Pensamiento y cultura estratégica en Suramérica: un análisis de sus libros blancos de defensa (pp. 89-119). Sello Editorial ESMIC.

Reynolds, P. (1977). Introducción al estudio de las Relaciones Internacionales. Editorial Tecnos.

Roth, A. (2002). Politicas Públicas. Formulación, implementación y evaluación. Edicones Aurora.

Zapata-Barrero, R. (2012). Teoría política de la frontera y la movilidad humana. Revista Española de Ciencia Politica, (29), 39-66. https://dialnet.unirioja.es/servlet/articulo?codigo $=4002502$ 